\title{
Fluorescence spectral diagnosis of malaria - a preliminary study
}

\author{
Vadivel Masilamani ${ }^{1,2^{*}}$, Sandhanasamy Devanesan², Mani Ravikumar ${ }^{3}$, Kantharaj Perinbam³, \\ Mohamad Saleh AlSalhi ${ }^{1,2}$, Saradh Prasad ${ }^{2}$, Siddanna Palled ${ }^{4}$, Kadirampatti Mani Ganesh ${ }^{4}$ \\ and Abbas Habeeb Alsaeed ${ }^{5}$
}

\begin{abstract}
Background: Malaria is the most common disease transmitted by the bite by an infected female anopheles mosquito and caused by the plasmodium parasite. It is mostly prevalent in subtropical regions receiving abundant rain and supporting copious mosquito breeding. This disease is generally detected by the microscopic examination of blood films or antigen based rapid diagnostic test. Only occasionally the parasite DNA is detected using polymerase chain reaction in certain advanced, expensive laboratories.

Methods: An innovative spectral detection method based on the fluorescence spectra of a set of blood plasma biomolecules [tyrosine, tryptophan, nicotinamide adenine dinucleotide (NAD), and flavin adenine dinucleotide (FAD)] and red blood cell (RBC)-associated porphyrin is being evolved by our group.

Results: The research so far has exhibited sensitivity and specificity values exceeding $90 \%$ based on the spectral features of blood components of 14 malaria patients and 20 numbers of age adjusted normal controls. The fluorescent biomolecules go out of proportion when the malarial parasite breaks down the hemoglobin of blood.
\end{abstract}

Conclusion: This technique has the potential to be used as an alternative diagnostic procedure for malaria since the instrumentation involved is portable and inexpensive.

Virtual Slides: The virtual slide(s) for this article can be found here: http://www.diagnosticpathology.diagnomx.eu/ vs/13000_2014_182

Keywords: Malaria diagnosis, Fluorescent biomarkers, Spectral diagnosis, Hemoglobin damage, Plasmodium falciparum

\section{Background}

Malaria is considered as one of the major health problems in subtropical countries like India and Bangladesh. It is a mosquito borne infectious disease, but caused by the parasite of the genus Plasmodium. The disease is transmitted through the bite of mosquito infected by the parasite. The mosquito bite introduces the malarial parasite from its saliva into the blood of the human host. The parasite travels to the liver to reproduce and mature up [1]. The common symptoms due to malaria include headache and fever,

\footnotetext{
* Correspondence: masila123@gmail.com

'Department of Physics and Astronomy, College of Science, King Saud

University, P.O Box 2455, Riyadh, Kingdom of Saudi Arabia

${ }^{2}$ Research Chair for Laser Diagnosis of Cancers, King Saud University, Riyadh, Kingdom of Saudi Arabia

Full list of author information is available at the end of the article
}

shivering, joint pain, convulsions etc., [2]. Malaria is a curable disease, if treated in earlier stages, but delay in treatment might result in death. The most common medications used for treating malaria include quinone (chloroquinone), artemisinin derivatives, in combination with mefloquine. Malaria is prevalent in the rainy season as the stagnant waters provide an adequate environment for the development of mosquito larvae. Since, there are no effective vaccines for malaria, the transmission of disease can be prevented by avoiding mosquito bites using repellents, nets and also applying insecticides on stagnant waters. [3].

When the sporozoites of the malaria parasites enter into the bloodstream through a mosquito bite, they migrate to the liver. The sporozoites infect the liver hepatocytes and get multiplied into merozoites asexually inside the hepatocytes (8-30 days). After maturing, they break the cell walls 
of hepatocytes and enter into the blood stream. Like the sporozoites, which multiplied inside the hepatocytes, the merozoites infect RBCs, get multiplied further within, finally breaking out of RBCs to invade fresh RBCs [4].

In humans, malaria is caused by five species of Plasmodium including Plasmodium falciparum, Plasmodium vivax, Plasmodium malariae, Plasmodium ovale and Plasmodium knowlesi. The three major human malaria species in India are Plasmodium falciparum, Plasmodium vivax and Plasmodium malariae [5].

Plasmodium falciparum is the most dangerous form of malaria with the highest rate of mortality. The treatment for malaria will be provided on the basis of the species identified upon standard diagnosis. In the states where Plasmodium falciparum and Plasmodium vivax co-exist, fluctuating proportions of the two species complicate the diagnosis and treatment process [5].

The diversity of malaria in India is also attributed through the increased diversity of malaria vector species. In India, of the 60 species of mosquito available, six were found to be the vector for malaria. Anopheles culicifacies with its indistinguishable sibling species: A, B, C, D, and, $\mathrm{E}$ is the major vector of malaria in India which accounts for $60-65 \%$ of the malaria burden. It also acts as a model organism to study the host-vector-parasite interactions [6].

Microscopy of stained thick and thin blood smears helps in the diagnosis of malaria. Their advantages include ease of use, inexpensiveness; but the major disadvantage is the moderate sensitivity (56-70\%) [7]. Rapid Diagnostic Tests such as antigen-antibody enzymatic analyses were developed to detect the circulating parasites and their antigens [8]. An advanced technique is available, that uses the PCR to detect the parasite's DNA, but is not widely used due to the high cost and complexity [6]. Some preliminary works have been done by others, employing spectroscopic techniques, based on UV- VIS absorption for monitoring malarial diseases $[9,10]$.

Spectral diagnosis is a newly evolving technique for detecting diseases, particularly cancer [11-16], thalassemia and sickle cell anemia [17]. This method is based on the difference in the concentration of fluorescent bio molecules, which indirectly acts as biomarkers for each type of disease. In continuation of our earlier works in this line, the paper presented here is a preliminary investigation to explore the possibility of diagnosing malaria by the spectral features of the infected blood.

\section{Methods}

The methods and materials for spectral diagnosis of cancer and hemoglobinopathies have been presented in a number of papers earlier [11-19]. The same procedure had been adopted here too. The spectral features of blood components [plasma and acetone extract of $\mathrm{RBC}$ ] were obtained from 14 persons [male 8 , female 6 with age ranging from $10-20$ ] diagnosed with malaria. In order to highlight the distinct differences, the spectral features of samples of normal control $[\mathrm{N}=20]$ have been presented and compared.

\section{Blood}

Controls: Exactly $5 \mathrm{ml}$ of venous blood from each of the 20 healthy volunteers (age range: 10 to 20 years) was collected in a violet sterile vial that contained the anticoagulant EDTA. The vial was gently rocked five times to adequately mix the EDTA and whole blood, and the samples was centrifuged at 3000 rotational speed (RPM) for 15 minutes. Clear, pale, greenish-yellow plasma supernatant was obtained by such centrifugation. A total of $1.5 \mathrm{ml}$ of supernatant was removed from the top layer for spectrofluorimetric analysis, leaving the buffy coat and the formed elements, as undisturbed sediment. The blood plasma sample was subjected to synchronous fluorescence excitation spectral analyses without any other treatment.

Next, the buffy coat, which contained mostly white blood cells (WBCs) (e.g., lymphocytes), was removed and discarded, and exactly $1 \mathrm{ml}$ of the thick formed elements from the bottom layer, which contained mostly RBCs, was removed to a sterile vial and mixed with $2 \mathrm{ml}$ of analytical grade acetone. Proper care was taken to ensure that the formed elements did not develop lumps. After thorough mixing to enable the acetone to extract fluorophores within and around the cells, the sample was centrifuged again (3000 rpm for 15 minutes). The resulting supernatant was subjected to fluorescence emission spectra analysis at an excitation wavelength of $400 \mathrm{~nm}$.

Patients: The same protocol was used to process blood samples from confirmed patients suffering from malaria caused by the parasite plasmodium falciparum as diagnosed by professional pathologists of General hospital, Chennai. Samples were obtained before any treatment. The patients were informed about the investigation, and proper consents were obtained. The samples were collected as per the ethical committee approval (GACN/ $\mathrm{KP} / \mathrm{CNBT} / 8428)$ The malaria subjects consisted of 14 patients (male 8, female 6 with age ranging from $10-$ 20 ), and had different levels of clinical severity.

\section{Instrumentation}

The instrument used was a spectrofluorometer (SL 174, Elico, India) capable of collecting excitation, emission, and synchronous spectra in the 200-800 nm range. An excitation and emission slit width of $10 \mathrm{~nm}$ and scan speeds of $1000 \mathrm{~nm} / \mathrm{min}$ were used. The samples were placed in quartz cuvettes and illuminated by a specified wavelength of light with a 10-nm spectral width and a spot size of $3 \times$ $2 \mathrm{~mm}$. The power at the point of illumination was approximately $20 \mu \mathrm{W}$, which was too low to induce photo 


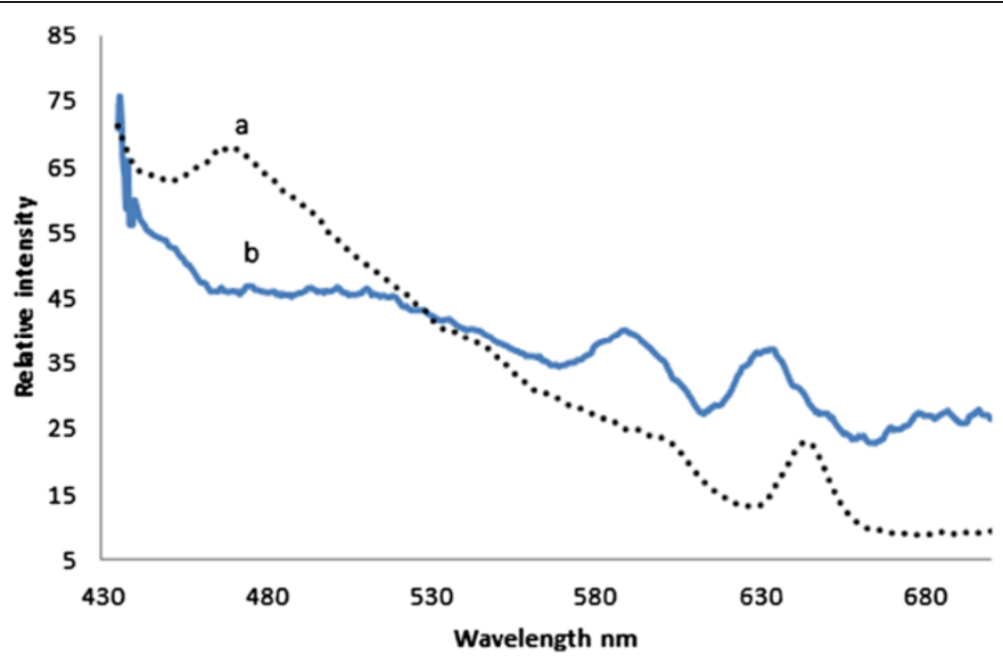

Figure 1 Fluorescence emission spectra (FES) of acetone extract of formed elements of (a) Normal control (b) Malaria patient.

bleaching. This finding was confirmed by repeating the experiment three times for each sample and observing no inter-replicate spectral differences.

Three types of spectra are measured in the field of fluorescence spectroscopy. In fluorescence emission spectra (FES), one particular wavelength is selected for the excitation of a molecule, and the fluorescence emission spectrum is obtained by rotating the emission grating over a predetermined range. The reverse is true of fluorescence excitation spectra (FXS), in which the peak emission band of a molecule is selected, and the excitation grating is rotated to scan the excitation spectra. In synchronous excitation spectra (SXS), both gratings are synchronously rotated at offsets of $40 \mathrm{~nm}$ or $70 \mathrm{~nm}$ to obtain the fluorescence excitation bands for every molecule in the predetermined range. The wavelength offset and scan range are not unique; they are determined empirically by trial and error, for a given set of experimental protocol. After analysing other offsets, including $10 \mathrm{~nm}$ and $30 \mathrm{~nm}$, it was determined that the $70 \mathrm{~nm}$ offset provided excellent resolution and good contrast between the normal and malaria samples because $70 \mathrm{~nm}$ is the Stokes shift [20] of the most important biofluorophores (e.g., tryptophan, nicotinamide adenine dinucleotide etc.) in blood plasma. Hence, all of the results presented for plasma were based on the synchronous excitation spectra (SXS). The blood plasma is a complex matrix, with many biofluorophores with overlapping absorption and emission bands. The biggest advantage of synchronous scan, with $\Delta 70 \mathrm{~nm}$ offset, is to limit the spectra with three distinct bands.

It is important to draw attention to the fact that concentration quenching and inner filter effect are inherent in

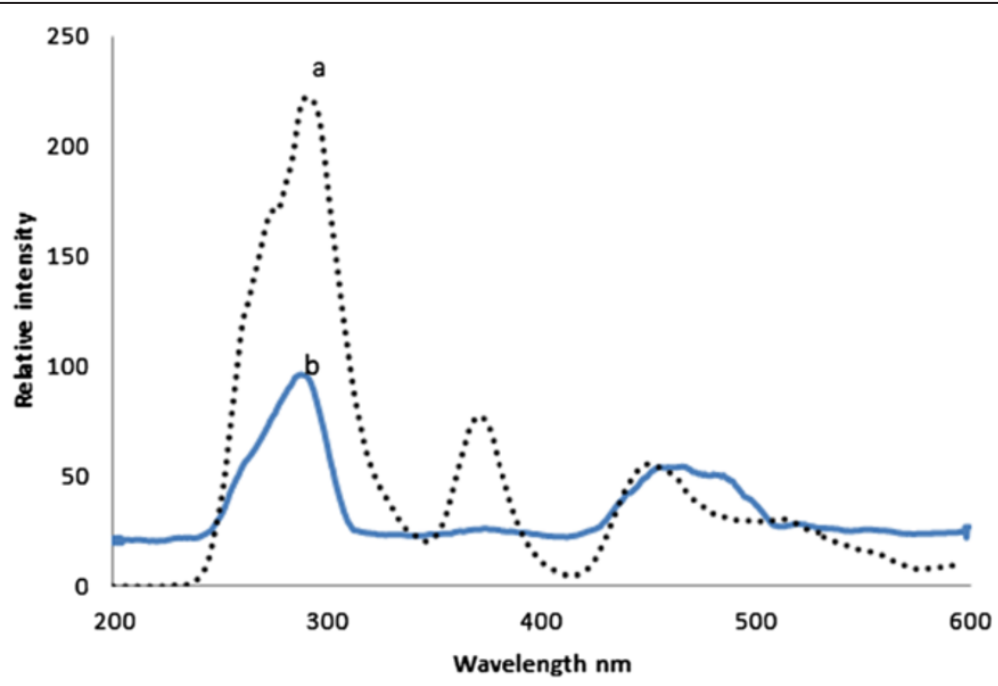

Figure 2 Synchronous excitation spectra (SXS) of blood plasma of (a) Normal Control (b) Malaria patient. 
such undiluted plasma. Hence the experiments were repeated for both sets of samples, after 5 times and 10 times serial dilution with normal saline. However, discrimination between the samples of patients and controls were best with undiluted samples and hence only this result was presented.

\section{Results}

Figure 1 (a), represents the typical fluorescence emission spectra (FES) of acetone extract of RBC of normal control (male, age 12). This spectra was obtained with the excitation wavelength fixed at $400 \mathrm{~nm}$ and emission scanned from 425 to $675 \mathrm{~nm}$.
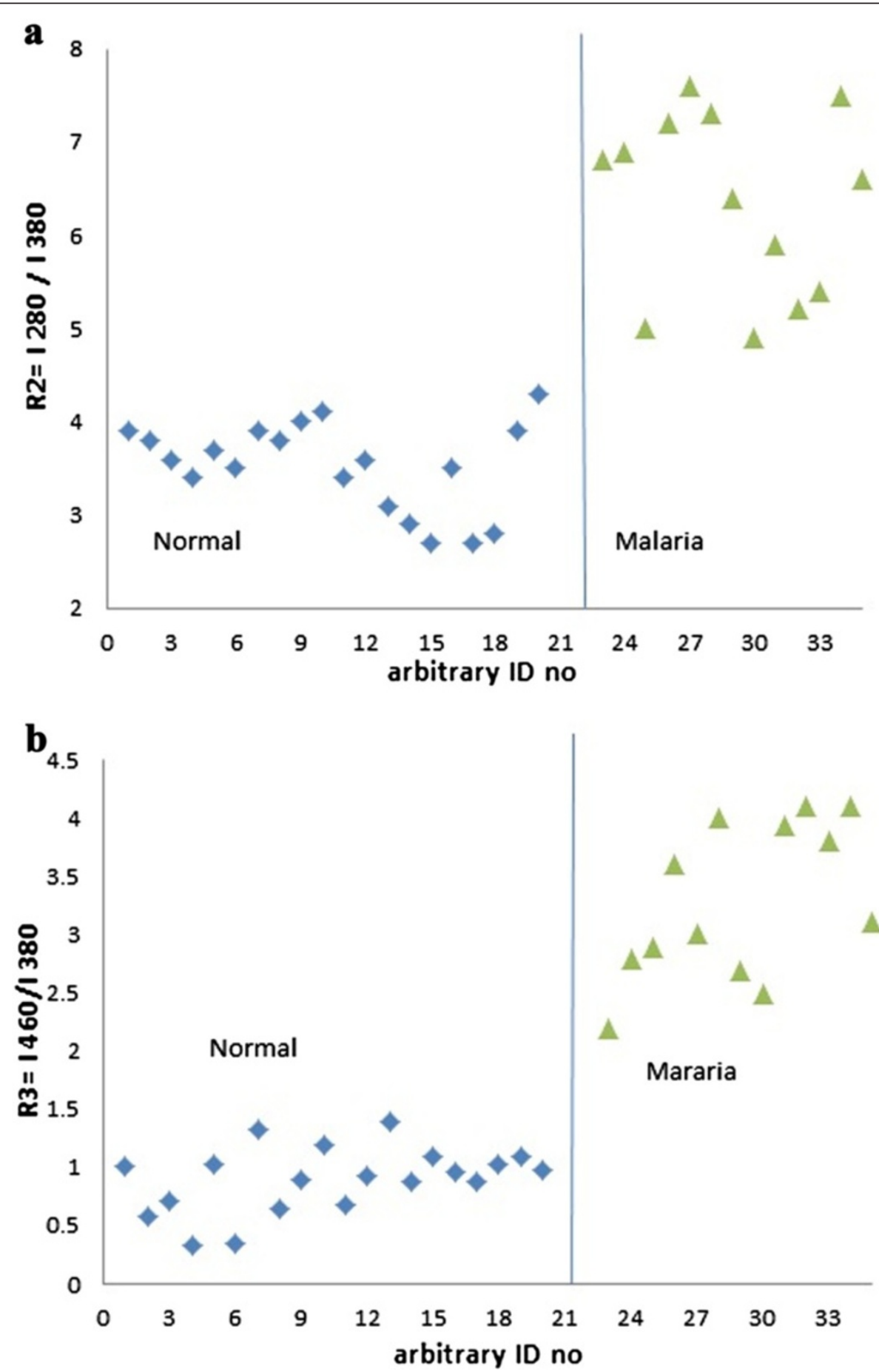

Figure 3 Discrimination between malaria and control samples. a) Scatter plot of R2 values (I 280/1380),obtained from SXS of plasma (b) Scatter plot of R3values (1460/1380), obtained from SXS of plasma. 
It could be seen that there were three important fluorescence bands, $460 \mathrm{~nm}$ (due to the metabolite flavin adenine dinucleotide, FAD), 585 (due to basic from of porphyrin, a protein found in hemoglobin), $635 \mathrm{~nm}$ (due to the neutral form of porphyrin). In fluorescence spectroscopy, the accepted convention is to take the ratio of peaks of fluorescence bands, in order to quantity and obtain a measure of endogenous biofluorophores. If we define the ratio $R_{1}=I_{635} / I_{585}$, the ratio of intensity of two fluorescence bands cited above, this was about $1.2 \pm$ 0.1 for normal control.

Figure 1 (b), represented the typical fluorescence spectra of acetone extract of RBC of a malaria patient (male age 18). The spectral features were similar to those of normal control, with the ratio $R_{1}=1.0 \pm 0.1$. That means, as far as porphyrin is taken as the biomarker, the difference between the malarial sample and normal control sample is only marginal. (The hump at $500 \mathrm{~nm}$, in the above spectrum was again due to FAD and this shift was not consistent and hence ignored).

Figure 2 (a) represented the SXS, the synchronous excitation spectra of normal blood plasma. It showed three main bands; at $280 \mathrm{~nm}$ (due to the excitation peak of the amino acid tryptophan); at $380 \mathrm{~nm}$ (due to co enzyme Nicotinamide adenine Dinucleotide, NADH); and at $460 \mathrm{~nm}$ (due to metabolite FAD). A ratio parameter $\mathrm{R}_{2}=$ $\mathrm{I}_{280} / \mathrm{I}_{380}$, (ratio of peak intensity at $280 \mathrm{~nm}$ and $380 \mathrm{~nm}$ ) was 4 for normal; another ratio parameter $R_{3}=I_{460} / I_{380}$ (ratio of peak intensity at $460 \mathrm{~nm}$ and $380 \mathrm{~nm}$ ) was about 1.0 for normal.

Figure 2(b) showed the SXS of blood plasma of the above malaria patient. This showed three main bands at $280 \mathrm{~nm}$, $380 \mathrm{~nm}$ and $460 \mathrm{~nm}$, (like those of normal plasma), but these bands were markedly out of proportion. For example, $R_{2}=6.9, R_{3}=3.2$ for the malarial sample. That is, $R_{2}$ was about $70 \%$ and $R_{3}$ was $300 \%$ elevated respectively.

a

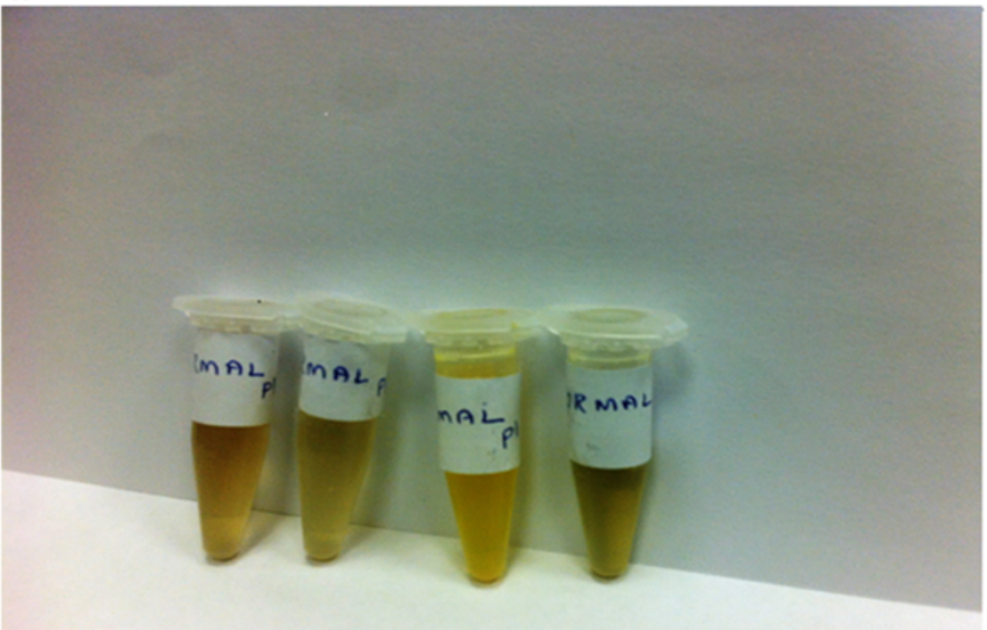

b

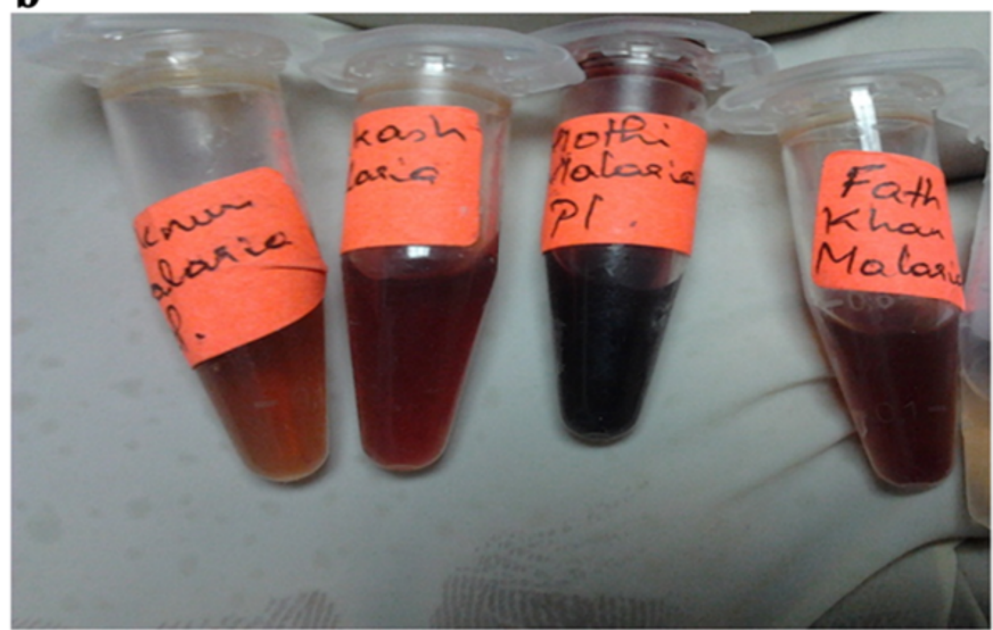

Figure 4 Photographs of blood plasma samples (a) of normal control (b) of malaria patient. 
Figure 3 (a) represented the scatter plot for $R_{2}$ and Figure 3 (b) for $R_{3}$ to classify the malaria samples from the control ones. The discrimination between the two sets was quite good.

\section{Discussion}

Spectral diagnosis is a newly evolving technique for detecting diseases, particularly cancer [11-16], thalassemia and sickle cell anemia [17]. It is based on the difference in the concentration of fluorescent biomolecules, which indirectly act as biomarkers. The same technique has been extended to the detection of malaria to explore the diagnostic potential since the instrumentation employed here was simple and inexpensive.

Out of the few diseases diagnosed by spectral features, it was thalassemia closest to the malaria, in terms of spectral manifestation. Thalassemia are inherited diseases due to reduced or defective $\alpha$ or $\beta$ chain of the hemoglobin $(\mathrm{Hb})$. Because of this, the concentration of RBC is only about 50 to $60 \%$ of the normal [17]. For example, the Hb level is $6 \mathrm{~g} / \mathrm{dL}$ for thalassemia patients; but for men, 13.5 to $17.5 \mathrm{~g} / \mathrm{dl}$, for women 12.0 to $15.5 \mathrm{~g} / \mathrm{dl}$ for normal controls. Such a decrease in RBC has manifested correspondingly in $R_{1}$ as shown in our earlier papers [17]. In comparison, for malaria patients, $R_{1}$ has decreased only marginally or from the window of $R_{1}$ the sensitivity and specificity of malaria diagnosis would be very poor.

Another important aspect of thalassemia is the poor average lifetime of $\mathrm{RBC}$, while the average lifetime for normal controls is about 120 days for the thalassemia patients it varied from 80 days to 10 days depending upon the severity. This is because the RBC of thalassemia patients is fragile. This is manifested as the abnormally high value of $R_{2}$ and $R_{3}$ as these two parameters area measure of metabolites of hemoglobin [17-19]. For example, $\mathrm{NADH}$ is abnormally low and FAD is high for thalassemia samples, because of which $R_{2}$ and $R_{3}$ is elevated. When $\mathrm{RBC}$ breaks down, a process that is abnormally high in thalassemia patients, the consumption of $\mathrm{NADH}$ is high, simultaneously producing equally abnormally high levels of FAD.

Similar degradation is externally initiated and perpetrated by the parasite which breeds and multiplies inside the RBC of malaria infected patients. In other words, malaria and thalassemia are similar from the point of view of the fragility of $\mathrm{RBC}$ the former being initiated by the parasite injection, and the latter being inherited. However, these two are dissimilar from the point of concentration of $\mathrm{RBC}$ as the former ones are persons with the same concentration of $\mathrm{RBC}$ as those of normal, but the latter ones are subjected with inherited low level of RBC.

Most of the symptoms and complications of malaria may be attributed to the rapid and abnormal lysis of the RBC's and this spectral diagnosis provide the direct evidence for this point of view. The photographs, presented in Figure 4 (a) for the plasma of normal and 4 (b) for patient, show the distinct difference. The pale reddish plasma of malarial patient is due to degradation of $\mathrm{RBC}$ induced by the parasite. In contrast the control plasma was pale greenish yellow.

\section{Conclusion}

In this preliminary study, done as a proof of concept, the fluorescence spectral features of blood components of malarial patients have been reported. The study shows that, the malarial patient sample could be distinguished with high sensitivity from the normal control, because plasma of a malaria patient is flooded with the fluorescent decay products of RBC. It is important to mention that this report presents results of only one type of malaria (PF). We need to repeat with other types of malaria to check possible distinction, a task difficult in the conventional technique. In addition, we need to do large-scale, multicenter study to assess the clinical viability of this new technique. We hope to undertake this task in the near future.

\section{Competing interests \\ The authors declare that they have no competing interests.}

\section{Authors' contributions}

V.M, S.D, M.S.A and S.P wrote the paper; and made a substantial contribution to the data analysis; M.R, K.P the collected the samples and did the experiment; S.P, K.M.G and A.S contributed in the hematological data analysis; All authors read and approved the final manuscript.

\section{Acknowledgements}

The authors would like to extend their sincere appreciation to the Deanship of Scientific Research at King Saud University for its funding of this research through the Research Group Project No "RGP- VPP-223".

\section{Author details}

'Department of Physics and Astronomy, College of Science, King Saud University, P.O Box 2455, Riyadh, Kingdom of Saudi Arabia. ${ }^{2}$ Research Chair for Laser Diagnosis of Cancers, King Saud University, Riyadh, Kingdom of Saudi Arabia. ${ }^{3} \mathrm{PG}$ and Research Department of Plant Biology and Biotechnology, Govt Arts College for Men, Nandanam, Chennai, India. ${ }^{4}$ Radiation Oncology Department, KMIO, Bangalore, India. ${ }^{5}$ College of Medical Sciences, King Saud University, Riyadh, Saudi Arabia.

Received: 26 June 2014 Accepted: 7 September 2014

Published online: 17 October 2014

\section{References}

1. Dash AP, Valecha N, Anvikar AR, Kumar A: Malaria in India: challenges and opportunities. J Biosci 2008, 33:583-592.

2. Goswami G, Singh OP, Nanda N, Raghavendra K, Gakhar SK, Subbarao SK: Identification of all members of the anopheles culicifacies complex using allele-specific polymerase chain reaction assays. Am J Trop Med Hyg 2006, 75:454-460.

3. Shah NK, Dhillon GPS, Dash AP, Arora U, Meshnick S, Valecha N: Antimalarial drug resistance of plasmodium falciparum in India: changes over time and space. Lancet Infect Dis 2011, 11:57-64.

4. Singh N, Kataria O, Singh MP: The changing dynamics of plasmodium vivax and P. Falciparum in central India: trends over a 27-year period (1975-2002). Vector Borne Zoonotic Dis 2004, 4:239-248.

5. Sharma SK, Tyagi PK, Padhan K, Upadhyay AK, Haque MA, Nanda N, Joshi H: Epidemiology of malaria transmission in forest and plain ecotype villages in Sundargarh district, Odisha, India. Trans $R$ Soc Trop Med Hyg 2006, 100:917-925. 
6. Singh V, Mishra N, Awasthi G, Dash AP, Das A: Why is it important to study malaria epidemiology in India? Trends Parasitol 2009, 25:452-457.

7. Lema OE, Carter JY, Nagelkerke N, Wangai NW, Kitenge P, Gikunda SM, Arube PA, Munafu CG, Materu SF, Adhiambo CA, Mukunza HK: Comparison of five methods of malaria detection in the outpatient setting. Am J Trop Med Hyg 1999, 60:177-182.

8. Joel CM, Dean Goldring JP: Malaria rapid diagnostic tests: challenges and prospects. J Med Microbiol 2013, 62:1491-1505.

9. Serebrennikova YM, Patel J, García-Rubio LH: Interpretation of the UV-visible spectra of malaria parasite plasmodium falciparum. Appl Opt 2010, 49:180-188.

10. Serebrennikova YM, Patel J, Milhous WK, García-Rubio LH: Quantitative analysis of morphological alterations in plasmodium falciparum infected red blood cells through theoretical interpretation of spectralmeasurements. J TheoretBiol 2010, 265:493-500.

11. Masilamani V, AIZhrani K, AISalhi MS, Al Diab A, AlAgeily M: Cancer diagnosis by autofluorescence of blood components. J Luminescence 2004, 109:143-154.

12. Kalaivani R, Masilamani V, Sivaji K, Elangovan M, Selvaraj V, Balamurugan SG, AlSalhi MS: Fluorescence spectra of blood components for breast cancer diagnosis. Photomed Laser Surg 2008, 26:251-256.

13. Masilamani V, Rabah D, AlSalhi MS, Trinka VM, Vijayaraghavan P: Spectral discrimination of benign and malignant prostate tissues - a preliminary report. Photochem Photobio 2011, 87:208-214.

14. AlSalhi MS, Masilamani V, Trinka VM, AINachawati A, VijayaRaghavan AP: Lung cancer detection by native fluorescence spectra of body fluid- a preliminary study. J Fluorescence 2011, 21:637-645.

15. AlSalhi MS, Ben AS, Farhat K, Rabah D, Devanesan S, Atif M, Masilamani V: Optical biopsy of breast cancer tissue. Laser Phy 2012, 22:1358-1363.

16. AlSalhi MS, AIMehmadi AM, Abdo AA, Prasad S, Masilamani V: Diagnosis of liver cancer and cirrhosis by the fluorescence spectra of blood and urine. Tech Can Res Treat 2012, 11:345-351.

17. Masilamani V, AlSalhi MS, Devanesan S, Farjah H, AlGahtani, AbuSalah KM, Ahamad I, Agastian P: Spectral detection of sickle cell anemia and thalassemia. Photodia PhotodynTher 2013, 10:429-433.

18. Devanesan S, AlSalhi MS, Ravikumar M, Perinbam K, Prasad S, Abbas HS, Palled SR, Jeyaprakash K, Masilamani V: Fluorescence spectral classification of iron deficiency anemia and thalassemia. J Biomed Opt 2014, 19:027008-027014.

19. AlSalhi MS, AlGahtani FH, Devanesan S, Trinka VM, Jeyaprakash K, AlSaeed AH, Masilamani V: Spectral detection of thalassemia: a preliminary study. J Biomed Sci 2014, 21:26

20. Alfano RR, Yang Y: Stokes shift emission spectroscopy of human tissue and key biomolecules. IEEE J QuanElec 2003, 9:148-153.

doi:10.1186/s13000-014-0182-z

Cite this article as: Masilamani et al:: Fluorescence spectral diagnosis of malaria - a preliminary study. Diagnostic Pathology 2014 19:182.

\section{Submit your next manuscript to BioMed Central and take full advantage of:}

- Convenient online submission

- Thorough peer review

- No space constraints or color figure charges

- Immediate publication on acceptance

- Inclusion in PubMed, CAS, Scopus and Google Scholar

- Research which is freely available for redistribution

Submit your manuscript at www.biomedcentral.com/submit
C Biomed Central 